\title{
Aspiration of extracted tooth under general anesthesia
}

\author{
Isaac Z Pugach and Sofya Pugach* \\ Complete Med care, Dallas, TX, USA
}

\begin{abstract}
This case report describes aspiration of extracted tooth under general anesthesia during routine dental care. Chest x- ray was done one week after dental treatment and foreign body $(\mathrm{FB})$ aspiration was discovered. We report this case to remind dental providers that early diagnosis of $\mathrm{FB}$ aspiration during routine dental care is crucial because prompt diagnosis of foreign body aspiration is key for prevention of complications.
\end{abstract}

\section{Introduction}

Aspiration of foreign body is serious and life -threatening complication of routine dental treatment. The risk of aspiration is significantly increased when patient is under general anesthesia in supine position. Foreign body aspiration can be fatal condition if aspirated object is large and causes complete obstruction of airway. According to the literature about 500-2000 deaths occur every year from foreign body aspiration in United States [1].

Usually early signs of aspiration of foreign body are cough, dyspnea, wheezing and stridor [2]. However, some small foreign body (tooth) aspirated may be remain asymptomatic for several months but can cause late complications such as post-obstractive pneumonia, pneumothorax, atelectasis, lung abscess, hemorrhage and even death $[1,3]$. Therefore, prevention and early diagnosis of FB aspiration is highly important in routine dental care. We report this case to remind dental providers that prompt diagnosis of foreign body aspiration during any dental procedure is crucial because it is the most important key for prevention of complications.

\section{Case report}

70-year-old white female with past medical history of diabetes, hypertension and CABG surgery came to our medical office with chief complaint of severe non-productive cough for several days. No fever, chills, or stridor. She had three teeth extracted and three teeth implants placed under general anesthesia last week in the dental office. During this routine dental care patient was in supine position and general anesthesia was induced with IV anesthetics. Immediately after dental procedure patient developed coughing, but dentist said that she "might have swollen one tooth in the stomach". Patient was sent home without any investigation. Patient denied any coughing or breathing problems prior to dental treatment.

On physical exam her vital signs were stable (HR: 64; RR: 16, BP: 140/90; T: 97.8; pulse ox: $94 \%$ ), no cyanosis but she had mild bilateral wheezing at lung bases. Rest of physical exam was unremarkable. Chest $\mathrm{x}$ - ray was performed (Figures 1 and 2). Anterior posterior and lateral chest radiograph showing triangle shape foreign body located in the right main stem bronchus. Foreign body looks like tooth crown or tooth cup. No any sign of bronchus obstruction. Rest of lung exam was normal.

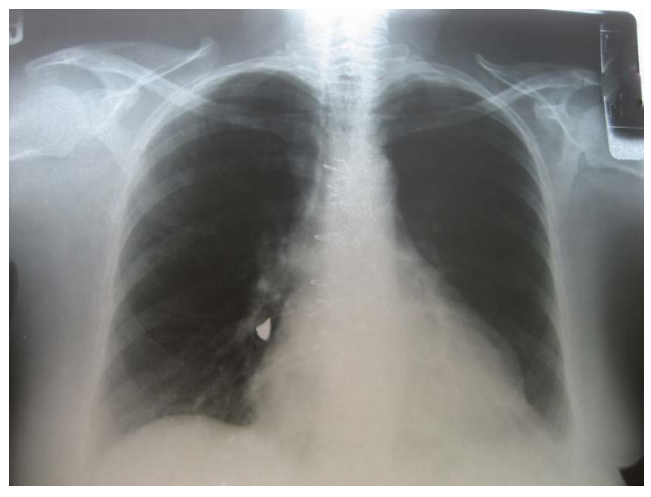

Figure 1. CXR anterior-posterior

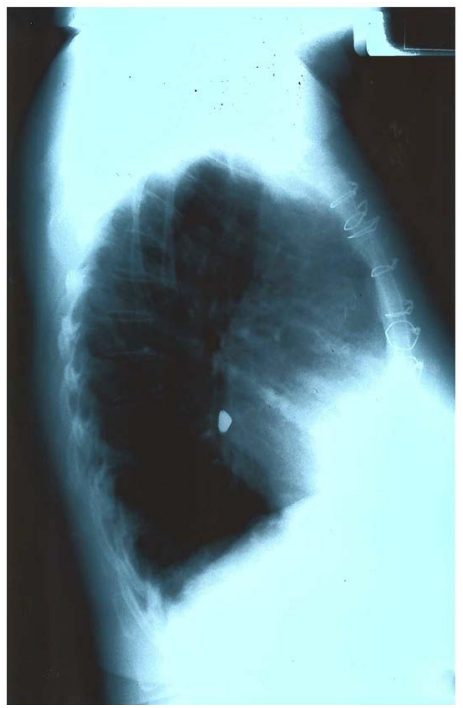

Figure 2. CXR lateral

${ }^{\star}$ Correspondence to: Sofya Pugach, Complete Med care, Dallas, TX, USA, E-mail: drpugach@yahoo.com

Key words: aspiration, ingestion, foreign body

Received: May 14, 2018; Accepted: May 25, 2018; Published: May 28, 2018 
Patient was diagnosed with Dx: Foreign body in right lung, low lobe and patient was immediately referred to the hospital for bronchoscopy to remove this aspirated tooth.

\section{Discussion}

Patients receiving routine dental treatment / procedure are at the risk that dental appliances/ teeth can be swallowing in GI tract due to their small size. Foreign bodies entering GI tract seldom represent serious medical condition, because they usually pass GI tract uneventfully. Only ingested a sharp and pointed objects can cause GI tract perforation and impaction. The common site of intestinal perforation is sigmoid colon and ileocecal junction. GI tract perforation occurs very rare (less than $1 \%$ ) and usually presents with abdominal pain, abdominal distention, nausea, vomiting and fever. These intestinal symptoms usually develop in 2-12 days after ingestion of FB [4].

However, foreign bodies entering the airway are serious and life -threatening complications of routine dental treatment. Dental providers should be extremely careful performing all dental procedures or handling small instruments during any intervention. The risk of aspiration is significantly increased when patient is under general anesthesia in supine position. Elderly, sedated patients, patients with neurological disabilities are main risk factors for foreign body aspiration in adults. Diagnosis of foreign body aspiration and its recovery should be done immediately to prevent patient morbidity and mortality $[1,4]$.

Complete blockage of airway is a medical emergency. Symptoms like acute choking with respiratory failure, cyanosis, loss of consciousness and brain injury can develop in several minutes if airway obstruction is not reduced [5]. Even these accidents occur rare, but it associated with high morbidity and even mortality. In emergency situation patient should be placed in to reverse Trendelenburg position and encourage coughing. If it fails to relieve airway obstruction, it is necessary to perform Heimlich maneuver, back slaps and abdominal thrusts. If these manipulations do not reduce the airway obstruction then this patient should have transferred to the hospital immediately $[6,7]$.

Therefore, prevention and early diagnosis of foreign body aspiration is highly important in routine dental care. Usually aspirated foreign body is lodging in the right main stem bronchus in adults because it is larger diameter and more vertical disposition than left bronchus [8]. Our case report had confirmed this statement, and foreign body was located in the right main bronchus. However, FB can be found in different airway location and different parts of the lung.

Typically, food (nuts, seeds) is the most common aspirated foreign body, but dental related appliances/tooth/crown/dental bridges represent up to twenty seven percent (27\%) of all aspirated cases [4]. The clinical presentation of foreign body aspiration depends from the site where this object had trapped.

The main symptoms of laringo-tracheal obstruction are dyspnea, cough and stridor. However, very small aspirated objects can pass through the vocal cords and not causing the obstruction of the upper airway and remain asymptomatic for many months [9]. Retention of this small object in the lung can result delay complications such as the vocal cord paralysis, post-obstructive pneumonia, atelectasis, bronchiectasis, pneumothorax, lung abscess, lung collapse, chronic pulmonary infection and even death $[3,10]$.

If there is a suspicious for foreign body aspiration in asymptomatic or symptomatic patients the posterior anterior and lateral chest $\mathrm{x}$ - ray is the first choice. There are no any problems or difficulties to detect tooth /metal objects in plain and lateral radiography. In some cases, chest $\mathrm{x}$ - ray can display secondary effects of aspiration in the lung such as mediastinal shift, obstructive emphysema, atelectasis and pneumonia. In our case the aspirated extracted tooth was very noticeable in both radiography views. When aspiration of a radiolucent material is suspected the diagnosis of FB aspiration is more difficult.

However, bronchoscopy should be done for exact diagnosis and retrieval of aspirated foreign body. According to the literature more than $95 \%$ of foreign body has been retrieved by bronchoscopy, but some cases required surgical procedures $[10,11]$. Cases where FB stays in the lung for more than 24 hours are associated with increased complications. Severe mucosal inflammation, irritation and nearby bronchial granulation tissue can hinder the removal of the foreign body. The earlier FB aspiration is diagnosed, the more easily it can be removed from the lung.

\section{Conclusion}

Dental providers play crucial role in preventing FB aspiration. If tooth/dental appliances cannot be found after extraction/intra oral manipulation the chest $\mathrm{x}$ - ray need to perform to confirm if tooth/ dental appliances were ingested or aspirated. Early diagnosis of foreign body aspiration during routine dental care is crucial because prompt diagnosis of foreign body aspiration is key for prevention of complications.

\section{References}

1. Mahmoud M, Imam S, Patel H, King M (2012) Foreign body aspiration of dental bridge in the left main stem bronchus. Case reports in Medicine 1-4.

2. Rakesh Kumar Y, Hemant Kumar Y, Chandra A, Yadav S, Verma P, et al. (2015) Accidental aspiration/ingestion of foreign bodies in dentistry: A clinical and legal perspective. Natl J Maxilofac Surg 6: 144-151. [Crossref]

3. Adelman HC (1988) Asphyxial deaths as a result of aspiration of dental appliances: A report of three cases. J Forensic Sci 33: 389-95. [Crossref]

4. Kumar U, Umesan, Chua KL, Balakrishnan P (2012) Prevention and management of accidental foreign body ingestion and aspiration in orthodontic practice. Ther Clin Risk Manag 8: 245-252. [Crossref]

5. Cameron SM, Whitlock WL, Tabor MS (1996) Foreign body aspiration in dentistry: a review. J Am Dent Assoc 127: 1224-1229. [Crossref]

6. Mann R, Srinivasan B, Baker A (2012) Managing aspiration. British Dental Journal $1-4$.

7. Warshawsky M (2015) Foreign body aspiration treatment \&management. Medscape $1-4$.

8. Baharloo F, Veyckemans F, Francis C, Biettlot MP, Rodenstein DO (1999) Tracheobronchial foreign bodies: presentation and management in children and adults. Chest 115:1357-1362. [Crossref]

9. Israel H, Leban S (1984) Aspiration of an endodontic instrument. J endod 10: 452-454

10. Ulku R, Baskan Z, Yavuz (2005) Open surgical approach for tooth aspirated during dental extraction: A case report. Aust Dent J 50:1 (49-50). [Crossref]

11. Kose A, Kostak D, Aramangan E, Durak A, Seçkin NS, et al. (2014) Tracheobronchial Foreign body aspiration: dental prosthesis. Case Rep Pulmonol 2014: 465856. [Crossref]

Copyright: (C2018 Pugach IZ. This is an open-access article distributed under the terms of the Creative Commons Attribution License, which permits unrestricted use, distribution, and reproduction in any medium, provided the original author and source are credited. 